\title{
Stigma among Veterans with urinary and fecal incontinence
}

\author{
${ }^{1,4}$ Andrew R. Devendorf, B.A.. ${ }^{2}$ Sarah Bradley, MA ${ }^{1}$, Lelia Barks, PhD, APRN, CRRN ${ }^{2}$, Angelina \\ Klanchar, RN, BSN, $\mathrm{MS}^{3}$, Tatiana Orozco, $\mathrm{PhD}^{1}$, Linda Cowan, PhD, APRN, FNP-BC, CWS ${ }^{1}$ \\ ${ }^{1}$ Tampa VA Research and Education Foundation, Inc., Tampa, FL; ${ }^{2}$ James A. Haley Veterans Hospital and Clinics, Tampa, \\ FL; ${ }^{3}$ North Florida South Georgia Veterans Health System, Gainesville, FL; ${ }^{4}$ University of South Florida, Department of \\ Psychology, Tampa, FL.
}

People with urinary incontinence or fecal incontinence experience elements of stigma, including embarrassment and shame. However, no study has investigated the lived experience of incontinence amongst Veterans or their caregivers. This study focuses on the qualitative data provided from 26 qualitative interviews conducted with 18 Veterans and 8 caregivers (including 4 dyads). Interviews were analyzed with a rapid-assessment matrix, using a systematic and iterative thematic analysis. Additionally, descriptive quantitative data was collected to triangulate the qualitative analysis. Four themes emerged that indicated strong salient experiences related to stigma: (1) Discussing incontinence (2) Education about incontinence (3) Social consequences and (4) Self-esteem. From both Veterans and caregivers' perspectives, self-stigma amongst Veterans emerged as embarrassment, shame, feelings of differentness, and a decreased self-esteem related to incontinence. Many patients even avoided labels associated with incontinence by using euphemisms to describe their symptoms, and caregivers indicated that Veterans were originally reluctant to disclose symptoms in an effort to hide their incontinence. These feelings appeared to contribute to Veterans' help-seeking behaviors. While our sample, by design, included Veterans who sought treatment for their incontinence, $48 \%$ waited over 1 year to speak to a healthcare provider, despite $50 \%$ reporting that incontinence was a "moderate" or "big" problem that altered daily activities. Our findings suggest healthcare providers working with Veterans with incontinence must consider the impact of incontinence and its associated stigma on mental health, and provides evidence that greater education for Veterans, caregivers, and health professionals is necessary for reducing stigma around the condition.

Corresponding Author: Andrew R. Devendorf, andrewdevendorf@gmail.com

Supplementary material: will be posted with final online publication. Please email andrewdevendorf@gmail.com if interested.

Acknowledgements: The investigators retained full independence in the conduct of this research.

Funding: This project was funded by a grant from DOMTAR Personal Care, Inc. through the Tampa VA Research and Education Foundation.

Disclaimer: The contents of this manuscript do not represent the views of the U.S Department of Veterans Affairs or the United States Government.

CAmerican Psychological Association, [2020]. This paper is not the copy of record and may not exactly replicate the authoritative document published in the APA journal. Please do not copy or cite without author's permission. The final article is available, upon publication, at: [ARTICLE DOI TO BE DETERMINED]

Keywords: Urinary incontinence; Fecal incontinence; Stigma; Veteran; Quality of life

Approximately 15 million adults experience urinary incontinence (UI), fecal incontinence (FI), or both, in the United States (Lukacz, SantiagoLastra, Albo, \& Brubaker, 2017; Markland et al.,
2010). Both UI and FI are associated with decreases in physical, mental, and social wellbeing (Chang, Gonzalez, Lau, \& Sier, 2008; Mardon, Halim, Pawlson, \& Haffer, 2006). 
Additionally, people with incontinence may experience significant stigma, given the embarrassment associated with bladder or bowel leaking (Dibley \& Norton, 2013). While effective treatments and management options exist, incontinence remains a severely under-reported and untreated condition (Harris, Link, Tennstedt, Kusek, \& McKinlay, 2005; Lukacz, et al., 2017), especially in the Veteran population (Smoger et al., 2000). Although there are a number of qualitative studies investigating the lived experience amongst patients with incontinence (for review, see Toye \& Barker, 2020), there are no qualitative studies investigating the experiences of Veterans with incontinence (or their caregivers), which would provide insight into (a) how incontinence impacts Veterans' (and caregivers) quality of life and (b) existing barriers that discourage Veterans from seeking and using treatments. This study seeks to understand the lived experiences of Veterans with UI or FI through a stigma framework.

\section{Stigma and Incontinence}

Individuals with incontinence may be especially vulnerable to experiencing public and self-stigma. Public stigma refers to stereotypes, prejudice, and discrimination that emerges when the general public endorses specific stereotypes (Goffman, 1963; Rüsch et al., 2005). For example, incontinence in the U.S. may be stereotyped as a symbol for aging (Mittness \& Barker, 1995), losing control over one's body (Elstad, Taubenberger, Botelho, \& Tennstedt, 2010), or as taboo topic (Ashworth \& Haggan, 1993). Consequently, prejudice in the form of negative attitudes (e.g., blame; Mitteness \& Wood, 1986) and emotions may occur toward people with incontinence. Negative emotions may include disgust, anger, frustration, and irritability (Yu et al., 1991; Toye \& Barker, 2020). When people endorse these stereotypes, or feel these emotions, they may behave differently toward people with incontinence, such as through social rejection and avoidance. For instance, in a qualitative study of spouses of non-Veteran patients with incontinence, some participants - despite being generally supportive and accepting of their spouse - reported avoiding intimate and sexual activities with the patient (Cassells, 2003).

The awareness of public stigma may lead patients to experience self-stigma, in which patients internalize the public's negative reactions and interpret stereotypes about incontinence as accurate. Self-stigma may invoke negative attitudes toward the self (e.g., "I am disgusting because I wet myself."), which may propagate social isolation (e.g., not talking to a spouse about symptoms) and avoidance of help-seeking behaviors (e.g., not seeing a healthcare provider; Wang, Wan, Wang, Kane, \& Wang, 2015). Indeed, incontinence has been referred to as a "silent affliction" (Leigh \& Turnberg, 1982), and in a review of UI studies, embarrassment and shame were found to be significant predictors of avoidance of help-seeking behaviors (Koch, 2006).

Qualitative work also documents how patients may experience fears related to the visibility of leaking, making frequent trips to the bathroom, and being unclean, which may increase feelings of shame and decrease help-seeking behaviors (Horroks, Somerset, Stoddart, \& Peters, 2004; Elstad et al., 2010; Taylor, Cahill, Rizk, 2014). Interestingly, Elstad et al. (2010) found gender and cultural differences in the perceptions of incontinence stigma, and how it relates to disclosing their condition. Women, for instance, feared being perceived as unclean and losing their social identity. Men, meanwhile, were afraid of being seen as impotent or lacking masculinity upon making multiple trips to the bathroom (e.g., "Males aren't supposed to go to the bathroom a lot; females, maybe, but not males."; Elstad et al., 2010, p. 2466). These findings may help explain discrepancies in help-seeking for men and women. In a community-based sample, $45 \%$ of women and $22 \%$ of men with weekly UI reported ever having sought care for UI (Harris, et al., 2005). Given these observed differences across groups, it is important to understand how different groups experience or perceive 
incontinence, and in particular within a Veteran population.

\section{Treatment Experiences for Patients with Incontinence}

Treatments and management options exist for UI, FI, and their symptoms (e.g., rashes). Options include medications (e.g.

anticholinergics/antimuscarinics, botox injections), behavioral treatments (e.g. pelvic floor muscle strengthening exercises, biofeedback, electrical-stimulation, physical therapy), surgery, powders, protective creams (e.g. zinc oxide), devices (e.g. condom catheters, urinary leg bags, pessaries, anal-vaginal balloon, etc.), and over-the-counter products (e.g., adult briefs/diapers, incontinence pads, odor neutralizers). However, patients may not query their healthcare providers about their incontinence to learn about these options. Reasons for not disclosing include shame, embarrassment, self-minimization of symptoms, lack of knowledge about incontinence, and low expectations for treatment (Horrocks et al., 2004; Taylor et al., 2014; Koch, 2006). Further, for patients who do seek treatment, there may be hesitation to using certain treatments, but this research area is relatively unexplored. For instance, patients may be recommended to wear feminine hygiene pads, but using this treatment may increase more feelings related to self-stigma (e.g., embarrassment, feeling weak). Thus, there is a need to understand how incontinence treatments also play a role in the experience of incontinence.

\section{Incontinence Amongst Veterans}

Few studies have explored UI or FI in Veterans, and preliminary findings about the prevalence of incontinence amongst Veterans are conflicting. From a study of three Veterans Health Administration (VHA) outpatient clinics, Smoger et al. (2000) found a UI prevalence rate among male Veterans at $32.3 \%$ (incontinence experienced at least once over past 12 months) and $13.8 \%$ (incontinence weekly). However,
Anger et al. (2008) found lower rates of UI among male $(.5 \%)$ and female $(2.2 \%)$ Veterans from outpatient clinics. Meanwhile, estimates of FI amongst Veterans are unknown, and there are no qualitative studies that have examined the experience of UI or FI in a Veteran population. These gaps in the literature are substantial, considering that military culture, values, and attitudes may impact Veterans' responses to incontinence, including via public and selfstigma.

For our purposes, culture refers to common ways of viewing the environment as it relates to values and priorities in life, including subconscious convictions, ideas, interpretations, and norms (Soeters, Winslow, \& Weibull, 2006). The U.S. military promotes a culture that values unit cohesion (the subordination of individual needs over the collective's needs), physical toughness or strength, the devotion to duty and the mission, and stoicism (e.g., emotional constraint) (Weiss $\&$ Coll, 2011). These values may impact military members' worldviews, or their perceptions and relationship with the world, which influence important decisions like seeking help, talking about problems, and putting themselves before others (Weis \& Coll, 2011).

These worldviews likely play important roles in the experiences of Veterans with incontinence. Given public perceptions that incontinence is linked with aging (Mittness \& Barker, 1995), loss of bodily control (Elstad et al., 2010), and not a serious illness (Hägglund, Walker-Engström, Larsson, \& Leppert, 2003), it is reasonable to expect that Veterans would be resistant to disclosing their problem, and, if they do disclose their problem, resistant to utilizing certain treatments. Further, the loss of bodily control and perception of aging may be especially distressful for Veterans, as it may symbolize loss of strength (or weakness). Altogether, Veterans may experience self-stigma and a decreased quality of life in the face of incontinence - even upon receiving treatments - but no study has investigated these questions. 


\section{The Current Study}

This study represents a formative investigation of lived experiences of Veterans who experience incontinence - either UI, or both UI and FI through semi-structured phone interviews. Data were drawn from a study about incontinence in Veterans from the perspective of the Veterans themselves and from caregivers of Veterans with incontinence. We analyzed the data using a stigma framework, meaning, we were interested in finding out if participants' experiences reflected themes evident in the stigma literature (Rüsch et al., 2005). Because the interviews were conducted to broadly assess Veterans' experiences, we developed hypotheses about the emerging themes as they would relate to incontinence and stigma. Based on available literature, we hypothesized that Veteran responses would incorporate themes of masculinity, embarrassment, perceived weakness, and not appraising incontinence as serious.

\section{Methods}

Data were drawn from a larger, mixed methods study (Cowan et al., in preparation), which was approved by the University of South Florida and James A. Haley VA Institutional Review Board (Pro 00038498), "Incontinence in Veterans At Home." The study employed a telephone survey of Veterans with incontinence and their caregivers (if the Veteran had a caregiver who assisted with their incontinence care and lived with them). A subset of participants completed semi-structured telephone interviews with questions about the experience of living with incontinence and of the caregiving of incontinence.

\section{Participants}

Recruitment targeted community-dwelling individuals aged $18-100$ years, who were contacted via mailing methods, referrals from physicians, brochures left in primary care clinics, and contact with the Veteran Engagement
Committee at the James A Haley VA (who provided valuable feedback and suggestions throughout the project). The larger study recruited male and female Veterans with fecal and/or urinary incontinence who met the following inclusion criteria: participants were able and willing to participate in a telephone survey, residing in either single family home or Medical Foster Home, and provided informed verbal consent. Caregivers were included if they lived with the Veteran and provided informed verbal consent. Exclusion criteria included the following: Veterans or their caregivers who did not agree to participate, did not speak English, or those with any disability that prevented them from communicating over the phone or giving their own verbal consent. These exclusions of communication did not prevent caregivers from participating in the study's caregiver portion.

Of 471 Veterans contacted, 275 did not respond, 73 did not meet inclusion criteria, 9 declined to participate, and 14 were lost to follow-up. In total, the larger study consisted of 64 Veterans with UI, FI, or mixed incontinence, and 36 caregivers (Cowan et al., in preparation). All participants were provided the opportunity to participate in an additional semi-structured telephone interview. Thus, this study focuses on the qualitative data provided by a convenience sample of 26 participants (18 Veterans, 8 caregivers) who completed semi-structured interviews.

\section{Procedure}

After taking a telephone survey, participants who agreed to complete the qualitative interview were contacted later and interviewed by Author \# 2 . Interview questions concerned participants' knowledge of incontinence, their experiences seeking treatment for incontinence, interactions with providers, symptom management, and satisfaction with incontinence products (see Qualitative Interview Guides in Supplement 
Material A and B). Interviews were recorded, transcribed verbatim, and checked for accuracy.

\section{Data Analysis}

Qualitative data analysis was primarily undertaken by Author \#2, with detailed consultation and interpretation from Author \#1. Quantitative analysis was conducted by Author \#5. Author \#1 was a doctoral student in clinical psychology with a background in qualitative methods and stigma surrounding chronic health conditions. Author \#2 was a doctoral candidate in anthropology with a background in qualitative methods and health inequities. Author \#1 is a research assistant and Author \#2 is a Medical Anthropologist at the James A Haley Veteran's Hospital and Clinics. Both are trained in and knowledgeable about military culture. The remaining authors (who provided additional guidance) were also employees of and had backgrounds in nursing, incontinence, and Veteran healthcare.

Given the authors' primary interest in understanding and describing Veterans' incontinence experiences as they relate to public and self-stigma (Rüsch et al., 2005), while also being open to other emerging themes, the authors analyzed the data using a combined deductive and inductive approach (Armat et al., 2018), with a constructivist-interpretivist lens. A constructivistinterpretivist perspective views reality as being constructed in the mind of the individual, rather than assuming there exists a single, objective reality (Ponterotto, 2005). This perspective is fitting for research about lived experiences as they relate to social phenomena like stigma. Because of the novel nature of the study (to our knowledge, this was the first qualitative study about Veterans and incontinence), the authors sought to provide a more descriptive account of Veterans' experiences with incontinence to help inform future work.

Prior to data analysis, Author \#1 and Author \#2 reviewed literature relevant to stigma, incontinence, and Veteran culture to become sensitive to underlying themes. Next, authors \#1 and \#2 collaboratively developed a matrix with definitions of stigma concepts (public, and selfstigma) and examples (of stereotypes, prejudice, discrimination, and behavioral consequences; see Appendix C for examples). Next, the data were analyzed using a Rapid Assessment Process.

The Rapid Assessment Process is an intensive, systematic and iterative analytical approach to efficiently identify emergent themes from qualitative data (Beebe, 2001). The analysis process included the following steps: (1) creation of domain names that correspond with interview and research questions (in order to organize participant responses); (2) development of a notetaking template using interview guide and domain names; (3) conduction of debriefings and refinement of notes following interviews and observations; (4) categorization of responses under corresponding domain names; (5) transfer of notes to an Excel® matrix; (6) review of categorizations and establishment of consensus among the team; and (7) analyzation and summarization of domains for key themes, variations, and information gaps, for example, as they related to, but not limited to, experiences of public and self-stigma (Rüsch et al., 2005). Emergent themes were reviewed by study team members, discussed from multiple points of view, and decided upon as a group to minimize risk of individual biases in interpretation.

\section{Results}

\section{Descriptive Results}

A total of 26 semi-structured qualitative interviews were conducted with 18 Veterans (nine male and nine female) and eight caregivers of Veterans with incontinence (all female). The Veteran sample is summarized in Table 1. Veteran participants were primarily white $(72 \%)$ and aged 50 and above $(89 \%)$. All Veterans had urinary incontinence (100\%) with some experiencing fecal incontinence in addition to the urinary incontinence (39\%). 
There were no Veterans who experienced only fecal incontinence without urinary incontinence.

Most participants had been experiencing incontinence symptoms for more than one year $(94 \%)$, but $45 \%$ waited over one year before seeking treatment for their symptoms. Many participants responded that they had changed aspects of their daily life due to their incontinence (61\%), considered incontinence to be a moderate or big problem for their social lives (50\%), and that incontinence limited their social activities $(61 \%)$. Eight participants were part of four Veteran/caregiver dyadic pairs.

Table 1: Veteran Participant Demographics $(N=18)$

\begin{tabular}{|c|c|c|}
\hline & $\mathbf{N}$ & $\%$ \\
\hline \multicolumn{3}{|l|}{ Incontinence } \\
\hline Urinary Incontinence & 18 & $100 \%$ \\
\hline Fecal Incontinence & 7 & $39 \%$ \\
\hline \multicolumn{3}{|l|}{ Gender } \\
\hline Male & 9 & $50 \%$ \\
\hline Female & 9 & $50 \%$ \\
\hline \multicolumn{3}{|l|}{ Age } \\
\hline $40-49$ & 2 & $11 \%$ \\
\hline $50-59$ & 5 & $28 \%$ \\
\hline $60-69$ & 6 & $33 \%$ \\
\hline $70-79$ & 5 & $28 \%$ \\
\hline \multicolumn{3}{|l|}{ Race } \\
\hline African American & 2 & $11 \%$ \\
\hline White/Caucasian & 13 & $72 \%$ \\
\hline Hispanic/Latino & 3 & $17 \%$ \\
\hline \multicolumn{3}{|l|}{ Employment } \\
\hline Employed & 4 & $22 \%$ \\
\hline Unemployed & 14 & $78 \%$ \\
\hline \multicolumn{3}{|c|}{ Total Time Experiencing Incontinence Symptoms } \\
\hline Less than 1 year & 1 & $6 \%$ \\
\hline $1-5$ years & 6 & $33 \%$ \\
\hline More than 5 years & 11 & $61 \%$ \\
\hline \multicolumn{3}{|c|}{ Has Sought Incontinence Treatment } \\
\hline Yes & 17 & $94 \%$ \\
\hline No & 1 & $6 \%$ \\
\hline \multicolumn{3}{|c|}{ Time Before Seeking Incontinence Treatment } \\
\hline Less than 6 months & 7 & $39 \%$ \\
\hline 6 months -1 year & 2 & $11 \%$ \\
\hline $1-5$ years & 5 & $28 \%$ \\
\hline More than 5 years & 3 & $17 \%$ \\
\hline N/A & 1 & $6 \%$ \\
\hline \multicolumn{3}{|c|}{ Do you change daily activities due to incontinence? } \\
\hline Never & 7 & $39 \%$ \\
\hline Rarely & 2 & $11 \%$ \\
\hline Sometimes & 6 & $33 \%$ \\
\hline Most of the time & 2 & $11 \%$ \\
\hline All of the time & 1 & $6 \%$ \\
\hline \multicolumn{3}{|c|}{ How big of a social problem is incontinence for you? } \\
\hline No problem & 4 & $22 \%$ \\
\hline Very small problem & 4 & $22 \%$ \\
\hline Small problem & 1 & $6 \%$ \\
\hline Moderate problem & 6 & $33 \%$ \\
\hline Big problem & 3 & $17 \%$ \\
\hline
\end{tabular}




\begin{tabular}{l|ll}
$\begin{array}{l}\text { How much of the time does incontinence limit social activi- } \\
\text { ties? }\end{array}$ & 7 & $39 \%$ \\
None of the time & 4 & $22 \%$ \\
A little of the time & 4 & $22 \%$ \\
Some of the time & 2 & $11 \%$ \\
Most of the time & 1 & $6 \%$ \\
\hline All of the time &
\end{tabular}

\section{Qualitative Results}

Qualitative data with Veterans and their caregivers revealed four primary themes related to Veteran and caregiver experiences with incontinence and associated stigma: 1) Discussing incontinence 2) Education about incontinence 3) Social consequences and 4) Self-esteem.

\section{Theme 1: Discussing incontinence}

When asked what words they used to describe their incontinence, the majority of participants used the term "incontinence," with nine participants explaining that they used euphemisms such as "leakage," "accident," "can't hold it," "weak bladder," "dripping," or "goosebumps" in place of using the term incontinence. Participants explained that discussing their incontinence was often difficult for them to do with both family members and healthcare professionals.

\section{Discussing incontinence with caregivers and family members}

Most caregivers explained they found out about the incontinence (of the Veteran they cared for) when doing laundry or when their Veteran had a public accident, not because the Veteran chose to discuss their symptoms. For example, one caregiver reflected that she first brought up the issue with her Veteran when she "was doing so much laundry and he was getting up a lot in the middle of the night and he was losing it [bladder control] on the floor, which made more work." Only one Veteran said that they spoke to a family member when they first began to experience incontinence; this was a male Veteran whose father was a primary care physician.
Some participants were continuing to hide their incontinence from loved ones at the time of interview. This included a female Veteran who did not want her husband to know about her incontinence, and a male Veteran who was hiding the issue from his two daughters. One female Veteran explained that she was "dumfounded that it was happening" to her when she first started experiencing symptoms, and she went and bought pads without revealing the issue to anyone.

Similarly, when asked why he was hiding the issue from his family, one male Veteran explained, "Well I had heard that people get teased. 'Oh look at that guy, he's got a wet spot on front of his pants.' And I actually experienced that teasing, so that's not much fun."

\section{Discussing incontinence with healthcare professionals}

Most participants saw their primary care healthcare provider for assistance with their incontinence symptoms, had positive experiences with their healthcare providers, and felt comfortable discussing their incontinence with their healthcare provider. One caregiver reflected that when she brought the issue up with her Veteran's healthcare provider, they "spent 45 minutes with the nurse going over everything," and even now during appointments, they "spend over an hour talking about incontinence: 'has it gotten better? has it gotten worse?"' Another caregiver explained, "Overall, they [VA] do a wonderful job. I can just call them and tell them what the problem is and they get right on it. They don't stop for nothing."

While most participants did feel comfortable when speaking to a healthcare provider about their incontinence, one male Veteran explained that, "I 
had a little trouble admitting it to female physicians or female nurses. It was too embarrassing," Similarly, one female Veteran said she felt uncomfortable seeking care from a male urologist because she found herself "being the only female in the waiting room in a sea of men" and "there was no female equipment in the treatment room". More than one third of participants described negative experiences they had with a healthcare provider when seeking incontinence care, because they were not taken seriously, no treatment alternatives were discussed, the healthcare provider was not listening, or the provider did not inquire about the topic. One male Veteran explained that, though he was comfortable talking about the issue, he was initially hesitant to discuss incontinence with his healthcare provider, and for that reason, "Maybe they [healthcare providers] should inquire about it as part of their list of questions. I had to bring up the whole thing when I was finally at my wits end as to what to do about it."

\section{Theme 2: Education about incontinence}

Both Veteran and caregiver participants referenced a lack of available information about incontinence, treatment, and products as representing a barrier to effective treatment. In some cases, participants also felt that the healthcare providers they were seeing were not sufficiently educated about the causes and treatments for incontinence.

\section{Veteran and caregiver knowledge about incontinence}

Before they began to experience symptoms, most participants knew little to no information about incontinence. Participants that had heard something about incontinence associated it with getting older, being a woman, or giving birth. When asked about when they first began to experience incontinence symptoms, several participants explained that when it first occurred, they did not know they were experiencing incontinence. This was the case for the female Veteran who reflected on receiving a UI diagnosis,
"It could be a good 10 years it's been going on, maybe even longer because it's been a long time. I just didn't associate it. I just thought it was just aging." This female Veteran learned that her incontinence was related to a hysterectomy she had a decade prior. She originally sought treatment for her issues that resulted in a hysterectomy but had not sought treatment for the UI that resulted from the hysterectomy. Other participants had learned from a healthcare professional that their incontinence was related to other health conditions after experiencing the condition for months or years, such as prostate conditions, back surgery, brain injury, bladder stones, and kidney disease.

\section{Healthcare professional knowledge about incontinence}

Regardless of their opinion of their overall experience discussing incontinence with their healthcare provider, a common discussion point was that Veterans had received inappropriate treatment recommendations for their incontinence. Several Veterans and caregivers said that they received recommendations that were medically inappropriate; for example, one caregiver recalled that her Veteran was given drugs that dehydrated him, and they had to discontinue using it because the Veteran was already prone to dehydration. A few participants specifically said that their treatment recommendations were too embarrassing for them to follow, such as the male Veteran who was recommend to use diaper briefs but found them too visible to others and preferred to use a condom catheter and urinary drainage bag.

Another male Veteran explained his negative experience seeking care while enlisted, saying, "Seems like in the military they don't take it seriously. They just basically tell you to deal with it." A common request was that healthcare providers ensure that they are well-versed in the variety of incontinence treatments that are available, and for whom they are appropriate. Participants who were pleased with the treatment recommendations they received were likely to reference the education they were given about their 
options, such as the caregiver who recalled, "I was very pleased because they were definitely open to trying different combinations of medications and time periods in order to allow us to explore our options."

\section{Theme 3: Social consequences of incontinence}

Many Veteran participants explained that their incontinence symptoms had changed the way that they interact with other people. Participants did not want others to be able to recognize that they were experiencing incontinence, either because the products they used were noticeable, or because they had an accident in public.

\section{Incontinence products}

Veterans and caregivers had specific complaints about the incontinence products that were available to them. The most common complaints were that products were too bulky and/or not available in the appropriate size. Other complaints included that products were too feminine, too saggy, did not control odor or did not absorb liquid. The bulkiness of products was especially of concern to female Veterans who explained that they always showed under women's clothing. One female Veteran explained that she felt, "They only have the really thick ones and they're giving those to men and to women even though women's clothes are so much smaller. It's hard to fit things under your clothes." One caregiver explained that her Veteran did not want to wear products because he was concerned that they did not control odor. She expressed, "I hate [the odor]. It's embarrassing for him. We can't do anything about the odor. There's still an odor even after changing and washing his clothes. We always pray that nobody knows what happened."

Veterans and caregivers were especially interested in products that made them feel "normal," like the female Veteran who described a TV commercial she saw: "I seen something like that on TV too. They said, 'I can be normal.' And she puts it, it looks like a diaper or something, on, and she breaks out dancing, and you can bend over. And I'm thinking, if I bend, the back might show if I don't have a long enough shirt on!" For many Veterans, feeling normal was associated with no one being able to ascertain that they were using incontinence products.

\section{Fear and self-isolation}

For a few participants, the unpredictability of their incontinence symptoms led to them experiencing a constant fear that they would have an accident while out of the house. A male Veteran explained his frustration by saying, "It's not fun. You're always on edge, afraid to fart...You're afraid to run water because you might just pee on yourself. It's not worth living like that." A female Veteran described a post-surgery experience when she said, "I lost 18 inches of my small intestine and it's caused a problem...I just live with constant diarrhea and it's made me incontinent. I can't, just walk across the room and you've got a problem. So, I wasn't even able to leave the house for seven months." For this Veteran, remaining homebound was preferable to risking having a public accident. Another female Veteran described her experiences by saying:

This incontinence thing...it's whipping me. It's really beating me down mentally. Because it's humiliating to be in a store and to have an accident. And people say, just put a diaper on, no problem. Well a) you've got to be able to afford them and b) I can't even wear underwear because I get severe yeast infections. And so, I'm worried about being chafed with those things. So I don't know, maybe I'm just being ridiculous. I know I have to use them.

The cost of incontinence products and her physical reaction to the diapers was preventing this Veteran from purchasing products that would manage her incontinence symptoms, despite the fact that not using products prevented her from daily activities like going to the store.

\section{Theme 4: Self-esteem in Veterans with}




\section{incontinence}

Participants made various connections between their experience of incontinence and the way that they viewed themselves. This included incompatibilities with both social and gender roles. Some participants had sought mental health treatment due to their incontinence.

\section{Gender and incontinence}

Several caregivers and male Veterans explained that they did not feel that products were sufficiently masculine or appropriate for men. Similarly, a female Veteran who was receiving treatment from a male urologist did not feel that female Veterans were getting appropriate treatment for their incontinence. One female Veteran, who had been hiding her incontinence from her husband for about a decade, said that it was important for her to do so in order to remain a sexually appealing woman for her husband. Explaining her relationship with her husband, this Veteran said, 'I'm a grown woman...He's not really aware of what I go through because I don't really discuss it with him. That would make me uncomfortable. Talking about that with my husband but not with my doctor." Products used to treat incontinence symptoms were also perceived to be gendered by several participants who referenced "men's underpants sizes" or described products as "frilly, like something you'd give a girl." One caregiver was sensitive to her Veteran's concerns about certain incontinence products when she explained, "Well, he's a guy. My husband's a retired Navy SEAL and he just, it's like a, I would say a pride issue. We generally don't have to use those [diapers]."

Some participants, primarily Male Veterans and their caregivers, were more likely to use language that described incontinence as a weakness in the body, such as the male Veteran who, when asked what we thought had caused his incontinence, said, "I guess due to my lack of control." Similarly, a caregiver of a male Veteran described her understanding of the issue by saying, "Incontinence to me was where the kidneys were weak.
It's lack of kidney control." The idea that incontinence represented a lack of control over the body was an ongoing theme.

\section{Perception of self}

A few Veteran participants discussed how their experience of incontinence had affected the way they see and understand themselves. Referencing the association between incontinence and old age, several Veterans said that they would not use certain products because it made them feel older to do so. One Veteran stated, 'I'm not using anything yet. I don't want to really, if you want to know the truth. It makes me feel like an old man." This Veteran's caregiver was also interviewed and had expressed frustration with this attitude, as she felt the Veteran often had accidents. Similarly, a female Veteran said, "Oh no, it's not diaper time yet! No. I'd wear three pads before I'd wear a diaper...I can't do that. To me, I'm not ready for that."

Two female Veteran participants explicitly mentioned seeing a mental health professional as a result of their incontinence. One of the women said, "My private life is important to me. I'm 46 years old and sometimes I feel like I'm in my 70 s due to the incontinence issues...I was recently diagnosed with depressive disorder based upon my medical condition. It does cause me to be depressed at times. So, I have to work really hard trying to overcome those feelings." Another female Veteran described:

This incontinence thing? I've got to tell you, mentally, it's breaking me down. I'm actually seeing a psychiatrist right now because this has just tore my life apart. I never thought I would be some 69-year-old woman, laying in a bed, messing her pants. That's not how I saw myself. I was always active, I was out in the community, making friends, going places, doing things. And now I don't go anywhere or do anything, I'm just like a lump. 
In both cases, the Veteran linked her mental health treatment to lifestyle changes she had to make due to incontinence symptoms.

\section{Discussion}

This is the first qualitative study to explore Veterans' experiences with incontinence. Given prior research on incontinence and military culture (e.g., Elstad et al., 2010; Weis \& Coll, 2011), we expected themes related to public and self-stigma to emerge. We hypothesized these themes would relate to Veterans' decisions to disclose their incontinence, seek treatment, and use treatment. Our findings generally support these hypotheses, while also providing insight into Veterans social and healthcare interactions.

From a public stigma standpoint, several Veterans and caregivers lacked knowledge about incontinence, but among participants familiar with incontinence, they associated incontinence with aging, being a woman, or giving birth. A few Veterans felt a sentiment that the military expects sufferers to "just deal with [incontinence].," which indicates stereotypes that seeking help may be a sign of weakness. These stereotypes are in line with previous research in civilian populations that conflates incontinence with a loss of control over one's body (e.g., Chelvanayagam, 2014; Elstad et al., 2010). However, future research should clarify the extent to which Veterans endorse this "weakness" stereotype in relation to incontinence. Overall, themes related to public stigma presented subtly, while themes related to self-stigma were more apparent.

From both Veterans and caregivers' perspectives, self-stigma amongst Veterans emerged as embarrassment, shame, feelings of differentness, and a decreased self-esteem related to incontinence. In interviews, some Veteran participants explained that they had stopped doing certain activities out of fear of "having an accident." This is similarly reflected in the survey results, wherein $17 \%$ of participants said that they had changed their daily activities due to incontinence most or all of the time, and an additional $33 \%$ changed their daily activities due to incontinence some of the time. Studies of incontinence among civilians have shown that the fear of incontinence being discovered can cause withdrawal from social participation in an effort to hide evidence of the condition (Chelvanayagam, 2014). This issue was evident in the qualitative interviews and survey data which showed $61 \%$ of participants said that incontinence limited their social activities, and 50\% said that incontinence represented a moderate or big social problem for them. Considering the high risk among the Veteran population for self-isolation (Demers, 2011), and associated mental health outcomes (Wilson, Hill, \& Kiernan, 2018), the way that incontinence can exacerbate individual withdrawal from social life requires further investigation.

Further self-stigma can be seen in the way that Veterans managed their incontinence. In the interviews, many patients avoided labels associated with incontinence altogether by using euphemisms to describe their symptoms, and caregivers indicated that Veterans were originally reluctant to disclose symptoms in an effort to hide their incontinence. These feelings appeared to affect Veterans' help-seeking behaviors. While our sample, by design, included Veterans who sought treatment for their incontinence, survey results showed that $48 \%$ waited over 1 year to speak to a healthcare provider, despite $50 \%$ reporting that incontinence was a "moderate" or "big" problem that altered daily activities. These findings are in line with other studies that have shown significant reluctance to consult healthcare practitioners about treatment options (Horrocks et al., 2004; Taylor et al., 2014). Fortunately, most Veterans felt comfortable talking about incontinence with their healthcare providers. Although, some Veterans encountered negative healthcare experiences like not being taken seriously or receiving unhelpful (or no) treatment recommendations. When Veterans did receive treatment recommendations, self-stigmatizing attitudes appeared to influence their willingness to use certain treatments or management tools, especially if they were no- 
ticeable, like bulky or odorous briefs. These findings are consistent with prior stigma research that documents how self-stigma relates to treatment behaviors (Corrigan \& Rao, 2012).

Our study adds to the preliminary literature that suggests cultural and gender differences in the experience of incontinence (Elstad et al., 2010) and illustrates perceptions about incontinence that may be unique to the Veteran population. In this sample, masculinity themes emerged amongst both male and female Veterans, and presented as Veterans not wanting to appear weak. These attitudes, along with felt shame and embarrassment, appeared to relate to Veterans preference for incontinence products that increased feelings of normalcy. However, male and female Veterans differed in some aspects of their experience.

Male Veterans expressed fears related to appearing as feminine, for instance, if they used certain products. One female Veteran expressed frustration about the dearth of female providers, and several felt less comfortable talking to men about incontinence. Some females also expressed fears about the sexual repercussions of disclosing their incontinence to their partner. Similar themes emerged in two qualitative syntheses of UI studies with women; UI may impair women's sexual functioning, intimacy, and sexual satisfaction, while increasing feelings of shame, blame, and guilt (Mendes, Hoga, Gongalves, Silva, Pereira, 2017). Incontinence may also increase sexual anxiety, threats to self-esteem, and feelings of being undesirable (Toye \& Barker, 2020).

In addition to self-stigma, our analysis suggests that cost and healthcare provider knowledge are barriers to incontinence treatment. Both Veterans and caregivers requested that providers become more literate about the variety of treatments, given that many Veterans were reluctant to use certain treatments or were recommended inappropriate treatments. Concurrently, healthcare providers may be in position to redress negative stereotypes, misconceptions, and other stigmatizing beliefs related to incontinence, while validating patients' experiences.

\section{Strengths, Limitations, and Future Research}

As one of the first qualitative investigations of the lived experiences of Veterans with incontinence, this study provides a foundation for understanding stigma in this population. A core strength of this study is its inclusion of both Veteran patient and caregiver perspectives, which allowed for a more comprehensive analysis of this sensitive and embarrassing topic. Our sample also included an even number of male and female Veterans, which allowed us to identify gender differences regarding self-stigma. Lastly, our qualitative findings triangulated with our descriptive quantitative findings. With these strengths, our study also has limitations.

First, our sample size is moderate for qualitative research (e.g., see Guest, Bunce, \& Johnson) and may not reflect the population of Veterans who experience incontinence as a whole. Future research might consider how other variables - including age, physical health status, and military branch - influence experiences with incontinence. Second, our sample was drawn from a larger study that included a convenience sample of Veterans who have sought incontinence treatment. This limitation prevented us from exploring how stigma might prevent Veterans from seeking incontinence care, although notably $48 \%$ of our sample waited at least one year to seek a healthcare provider. As well, including patients who sought treatment may explain our findings that, upon initiating treatment, many Veterans felt comfortable talking to healthcare providers. It is possible that our sample neglects the voice of Veterans who did not feel comfortable talking with a provider and are no longer in care. Third, our study was unable to recruit any male caregivers of Veterans with incontinence, and thus, we recommend future research seek to incorporate this valuable perspective. Similarly, future research is this area would also benefit from the perspective of healthcare workers providing treatments for incontinence to better understand how and why they recommend certain incontinence 
products and treatments to certain patients. Overall, greater study is needed on Veterans' experiences with incontinence, stigma, and healthcare, as this area remains understudied. We hope this qualitative study serves as a first step to inform a more in-depth exploration of Veterans' experiences using additional qualitative (e.g., grounded theory; ethnography) and quantitative approaches (e.g., longitudinal designs).

\section{Implications and Conclusion}

Male and female Veterans experience unique self-stigma related to incontinence, which may influence their willingness to disclose their problem to their caregivers, lead to delays in seeking treatment, and affect their attitudes toward using certain treatment. To improve incontinence for Veterans, we recommend that healthcare providers especially at the Veteran's Administration (VA) be equipped, knowledgeable, and ready to screen patients for UI and FI.

Additionally, as health gatekeepers, providers are in a position to alleviate stigma surrounding incontinence. However, research is needed to explore appropriate avenues to decrease incontinence stigma, given the complexities associated with health framing and stigma (e.g., see Corrigan, 2016 for lessons learned about erasing stigma). Our findings, for example, suggest that Veterans were resistant to using noticeable incontinence products due to fears of feeling embarrassed, regardless of the product's effectiveness for treating the Veteran's symptoms. On one hand, this finding suggests that providers should be more aware of concealable incontinence products. On the other hand, these fears of embarrassment suggest the existence of public stigma at a larger level and, perhaps long-term, there should be more public efforts to increase acceptance of people with incontinence. Ultimately, more awareness about knowledge, experiences, and treatments for incontinence, especially in macro systems like the VA, can improve quality of life for patients with incontinence.

\section{References}

Anger, J. T., Saigal, C. S., Wang, M., Yano, E. M., \& Urologic Diseases in America Project. (2008). Urologic disease burden in the United States: Veteran users of Department of Veterans Affairs healthcare. Urology, 72(1), 37-41.

Armat, M. R., Assarroudi, A., Rad, M., Sharifi, H., \& Heydari, A. (2018). Inductive and deductive: Ambiguous labels in qualitative content analysis. The Qualitative Report, 23(1), 219-221.

Ashworth, P. D., \& Hagan, M. T. (1993). The meaning of incontinence: A qualitative study of non-geriatric urinary incontinence sufferers. Journal of Advanced Nursing, 18(9), 14151423.

Beebe, J. (2001). Rapid assessment process: An introduction: AltaMira Press.

Bliss, D. Z., Norton, C., \& Vodusek, D. B. (2010). Raising awareness about fecal incontinence. Neurourology and Urodynamics: Official Journal of the International Continence Society, 29(4), 612-615.

Cassells, C., \& Watt, E. (2003). The impact of incontinence on older spousal caregivers. Journal of Advanced Nursing, 42(6), 607-616.

Chang, C. H., Gonzalez, C. M., Lau, D. T., \& Sier, H. C. (2008). Urinary incontinence and self-reported health among the U.S. Medicare managed care beneficiaries. Journal of Aging and Health, 20(4), 405-419.

Chelvanayagam, S. (2014). Stigma, taboos, and altered bowel function. Gastrointestinal Nursing, 12(1), 16-22.

Corrigan, P. W. (2016). Lessons learned from unintended consequences about erasing the stigma of mental illness. World Psychiatry, 15(1), 67-73.

Corrigan, P. W., \& Rao, D. (2012). On the self-stigma of mental illness: Stages, disclosure, and strategies for change. The Canadian Journal of Psychiatry, 57(8), 464-469.

Cowan et al.. (in preparation). Understanding incontinence in Veterans at home: A mixed-methods study

Demers, A. (2011). When veterans return: The role of community in reintegration. Journal of Loss and Trauma, 16(2), 160-179.

Dibley, L., \& Norton, C. (2013). Experiences of fecal incontinence in people with inflammatory bowel disease: self-reported experiences among a community sample. Inflammatory Bowel Diseases, 19(7), 1450-1462.

Elstad, E. A., Taubenberger, S. P., Botelho, E. M., \& Tennstedt, S. L. (2010). Beyond incontinence: The stigma of other urinary symptoms. Journal of Advanced Nursing, 66(11), 2460-2470.

Goffman E. (1963). Stigma: Notes on the management of spoiled identity. Englewood Cliffs, NJ: Prentice Hall

Guest, G., Bunce, A., \& Johnson, L. (2006). How many interviews are enough? An experiment with data saturation and variability. Field Methods, 18(1), 59-82.

Hägglund, D., Walker-Engström, M. L., Larsson, G., \& Leppert, J. (2003). Reasons why women with long-term urinary incontinence do not seek professional help: A cross-sectional population-based cohort study. International Urogynecology Journal, 14(5), 296-304.

Harris, S. S., Link, C. L., Tennstedt, S. L., Kusek, J. W., \& McKinlay, J. B. (2007). Care seeking and treatment for urinary incontinence in a diverse population. The Journal of Urology, 177(2), 680-684.

Horrocks, S., Somerset, M., Stoddart, H., \& Peters, T. J. (2004). What prevents older people from seeking treatment for urinary incontinence? A qualitative exploration of barriers to the use of community continence services. Family Practice, 21(6), 689-696. 
Koch, L. H. (2006). Help-seeking behaviors of women with urinary incontinence: An integrative literature review. Journal of Midwifery \& Women's Health, 51(6), e39-e44.

Leigh, R., \& Turnberg, L. (1982). Faecal incontinence: The unvoiced symptom. The Lancet, 319(8285), 1349-1351.

Lukacz, E. S., Santiago-Lastra, Y., Albo, M. E., \& Brubaker, L. (2017). Urinary incontinence in women: A review. JAMA, 318(16), 1592-1604.

Mardon, R. E., Halim, S., Pawlson, L. G., \& Haffer, S. C. (2006). Management of urinary incontinence in Medicare managed care beneficiaries: Results from the 2004 Medicare Health Outcomes Survey. Archives of Internal Medicine, 166(10), $1128-1133$.

Markland, A. D., Goode, P. S., Burgio, K. L., Redden, D. T., Richter, H. E., Sawyer, P., \& Allman, R. M. (2010). Incidence and risk factors for fecal incontinence in black and white older adults: A population-based study. Journal of the American Geriatrics Society, 58(7), 1341-1346.

Mendes, A., Hoga, L., Gonçalves, B., Silva, P., \& Pereira, P. (2017). Adult women's experiences of urinary incontinence: A systematic review of qualitative evidence. JBI database of systematic reviews and implementation reports, 15(5), 13501408.

Mitteness, L. S., \& Wood, S. J. (1986). Social workers' responses to incontinence, confusion and mobility impairments in frail elderly clients. Journal of Gerontological Social Work, 9(3), 63-78.

Mitteness, L. S., \& Barker, J. C. (1995). Stigmatizing a "normal" condition: Urinary incontinence in late life. Medical Anthropology Quarterly, 9(2), 188-210.

Ponterotto, J. G. (2005). Qualitative research in counseling psychology: A primer on research paradigms and philosophy of science. Journal of Counseling Psychology, 52(2), 126.

Rüsch, N., Angermeyer, M. C., \& Corrigan, P. W. (2005). Mental illness stigma: Concepts, consequences, and initiatives to reduce stigma. European psychiatry, 20(8), 529-539.

Siddiqui, N. Y., Levin, P. J., Phadtare, A., Pietrobon, R., \& Ammarell, N. (2014). Perceptions about female urinary incontinence: A systematic review. International Urogynecology Journal, 25(7), 863-871.

Smoger SH, Felice TL, Kloecker GH. Urinary incontinence among male veterans receiving care in primary care clinics. Annals of Internal Medicine. 2000;132(7):547-551.

Soeters, J. L., Winslow, D. J., \& Weibull, A. (2006). Military culture. In Handbook of the Sociology of the Military (pp. 237254). Springer, Boston, MA.

Taylor, D. W., Cahill, J. J., \& Rizk, D. E. (2014). Denial, shame and acceptance: Generating base-line knowledge and understanding of fecal incontinence amongst long-term care residents and care providers. Public Health Research, 4, 13-8.

Toye, F., \& Barker, K. L. (2020). A meta-ethnography to understand the experience of living with urinary incontinence: 'Is it just part and parcel of life?'. BMC Urology, 20(1), 1-25.

Wang, C., Li, J., Wan, X., Wang, X., Kane, R. L., \& Wang, K. (2015). Effects of stigma on Chinese women's attitudes towards seeking treatment for urinary incontinence. Journal of Clinical Nursing, 24(7-8), 1112-1121.

Weiss, E., \& Coll, J. E. (2011). The influence of military culture and veteran worldviews on mental health treatment: Practice implications for combat veteran help-seeking and wellness. International Journal of Health, Wellness \& Society, 1(2), 75-86.

Wilson, G., Hill, M., \& Kiernan, M. D. (2018). Loneliness and social isolation of military veterans: Systematic narrative review. Occupational Medicine, 68(9), 600-609.
Yu, C. Y., Johnson, K., Kaltreider, D. L., wei Hu, T., Brannon, D., \& Ory, M. (1991). Urinary incontinence: Nursing home staff reaction toward residents. Journal of Gerontological Nursing, 17(11), 34-41. 